\title{
Two-model Monte Carlo Simulation of Fire Scenarios
}

\author{
SIMO HOSTIKKA, TIMO KORHONEN, and OLAVI KESKI-RAHKONEN \\ VTT Technical Research Centre of Finland \\ P.O. Box 1803 \\ FI-02044 VTT, Finland
}

\begin{abstract}
A risk analysis tool called Probabilistic Fire Simulator (PFS) is developed for the computation of the distributions of fire model output variables and the sensitivities of the output variables to the inputs. PFS performs a Monte Carlo simulation using different fire models, including CFAST two-zone model and FDS fluid dynamics model. In this work, a new technique is developed for the use of two different fire models in the same Monte Carlo simulation. The two-model Monte Carlo technique provides a computationally effective means to improve the accuracy of the fast but inaccurate models, using the results of the more accurate but computationally more demanding models. The technique is tested in three scenarios: approximation of an analytical function, calculation of a ceiling jet temperature and a simulation of a simple room fire.
\end{abstract}

KEYWORDS: fire modelling, Monte Carlo simulation, risk assessment

\section{INTRODUCTION}

The numerical simulation of fires is widely used in the fire safety design and the risk analysis of large targets like shopping centres and industrial facilities. The uncertainty or the distribution of the predictions is of major interest in both applications. Presuming the model is principally valid for the given problem; the uncertainty of the prediction depends on, how the uncertainties of the input values are transferred through the system described by the model. In practically all applications, some uncertainty is related to the input values of the simulation. This uncertainty may be caused by the lack on information on the actual conditions, or by the fact that the simulation should actually represent a variety of different scenarios. Traditionally, the uncertainty has been taken into account by manually varying the input values. If more complete information of the output distribution is needed, Monte Carlo techniques should be used. This applies specially to fire problems, where many of the input distributions are skewed, of the class of lognormal distributions. In Monte Carlo, a large number of samples is randomly chosen from the input space and mapped through the system into the target distribution. Although Monte Carlo as a technique is almost sixty years old [1], its use in fire simulations has been prohibitively expensive. With modern computers, the situation has changed, and the tools described here have already been applied to engineering problems.

The numerical simulation of the complicated physical processes is always trading between the desired accuracy of the results and the computational time required. Quite often, the same problem can be tackled by many different models with different physical and numerical simplifications. A good example of this is the fire simulation, where zone models provide a fast way to simulate the essential processes of the fire, being inevitably coarse in the physical resolution. As an alternative, computational fluid dynamics (CFD) models have higher physical resolution and can describe more complicated physical 
processes. On the other hand, the time needed for the computation may be longer with several orders of magnitude. A technique is therefore needed, which can combine the results of the different models in a computationally effective way. A new technique, based on an intuitive approach, is proposed here. The technique allows the use of two different models in one Monte Carlo simulation, and is therefore called two-model Monte Carlo (TMMC). The technique is based on the assumption that the ratio of the results given by two models has smooth variations when moving from point to point of the random space. Therefore, if one of the models is presumably more accurate than the other, the ratio calculated at some point of the random space can be used to scale the result of the less accurate model within the neighbourhood of the point. The method is first presented in one dimensional problem, and extended later to general case.

In the previous research projects concerning the fire safety of Finnish nuclear power plants, a Monte Carlo tool called Probabilistic Fire Simulator (PFS) was developed [2]. The tool has been applied to fires in a cable tunnel and an electronics room. The tool allows the simulation of fire scenarios using various fire models, including two-zone model CFAST [3] and Fire Dynamics Simulator (FDS) [4]. The main outcomes of the tool are the distributions of the selected result variables, for example component failure time, and the sensitivities of the output variables to the input variables, in terms of the rank order correlations. The use of the rank order correlations allows the user to simultaneously identify both the modelling parameters and the actual physical properties that have the most influence on the results. The PFS tool has been implemented as a Microsoft Excel workbook with additional function libraries for the generation of random numbers and input and output of the external fire models. In the current work, the TMMC method is tested in three scenarios: approximation of an analytical test function, prediction of the ceiling jet temperature and a simple one room environment, where the CFAST results are scaled with the FDS results, and then compared against benchmark result, obtained by performing a full Monte Carlo with FDS. The validity of the used models is not discussed here. We do not compare our results with experiments either, because the results from some 1000 random experiments are not available for any real fire scenario.

\section{MONTE CARLO SIMULATION}

\section{General}

During the probabilistic safety assessment, one typically needs to estimate the probability that a certain component or system is damaged during a fire. This probability is a function of all possible factors affecting the development of the fire and the systems reaction to it. The occurrence of the target event is indicated by a limit state function, $g(t, \mathbf{x})$, which depends on time $t$ and a vector of random variables $\mathbf{x}$. As an example of the target event, we consider the damage of a component. The limit state condition is now defined using the function $g(t, \mathbf{x})$ :

$g(t, \mathbf{x}) \leq 0$, if the component is damaged at time $t$

$g(t, \mathbf{x})>0$, if the component is not damaged at time $t$ 
Other possible target events are, for example, heat detector activation and smoke filling. The development of a fire and the response of the components under consideration are assumed to be fully deterministic processes where the same initial and boundary conditions always lead to the same final state. With this assumption, the probability of an event at time $t$ can now be calculated by the integral

$$
P(t)=\iint_{\{\mathbf{x} \mid g(t, \mathbf{x}) \leq 0\}} \cdots \int_{X} \phi_{X}(\mathbf{x}) d x_{i}
$$

where $\phi_{x}$ is the joint density function of the random variables. In this work, the probability $P(t)$ is calculated using Monte Carlo simulation where input variables are sampled randomly from the given distributions. Latin Hypercube sampling [5] is used to generate samples from all ranges of the possible values, thus giving insight into the tails of the probability distributions.

The sensitivity of the output $y$ to the different input variables $x$ is studied by calculating the Spearman's rank order correlation coefficients (RCC). A value's “rank" is determined by its position within the min-max range of possible values for the variable. RCC is then calculated as

$$
\mathrm{RCC}=1-\frac{6 \sum d^{2}}{m\left(m^{2}-1\right)}
$$

where $d$ is the difference between ranks of the corresponding $x$ and $y$, and $m$ is the number of data pairs. RCC is independent of the distribution of the initial variable. The significance of the RCC values is studied with the methods of statistical testing. In small data sets, the actual values of RCC should be interpreted with caution due to the possible spurious correlations inside the input data [6].

\section{Two-model Monte Carlo (TMMC)}

We assume that we have two numerical models A and B, which can calculate physical quantity $a(x, t)$ depending on a parameter $x$ and the time $t$. In our analysis, $x$ is considered to be a random variable from a random space $\Omega$. The model B is more accurate than the model A, but the execution time of model B is longer than model A. The models are used to get two estimates of the time series: $\tilde{a}^{A}(x, t)$ and $\tilde{a}^{B}(x, t)$. The developed two-model Monte Carlo (TMMC) technique is based on the assumption that the results of the model A, at any point $x$ of the random space, can be corrected by multiplying them with scaling function, which is the ratio of model B time series to model A time series at some point $x_{s}$ in the vicinity of the current point $x$. The points $x_{s}$ are called scaling points.

In the beginning of the simulation, the random space is divided into distinct regions. Scaling function is then calculated for each region

$$
\Phi\left(\mathbf{x}_{s}, t\right)=\frac{\tilde{a}^{B}\left(\mathbf{x}_{s}, t\right)}{\tilde{a}^{A}\left(\mathbf{x}_{s}, t\right)}
$$


where $\mathbf{x}_{s}$ is the mid-point of the scaling region $\Omega_{s}$. During the Monte Carlo, the result of the model A is multiplied by the scaling function corresponding to the closest scaling point, to get the corrected times series $\tilde{a}^{A B}(\mathbf{x}, t)$

$\tilde{a}^{A B}(\mathbf{x}, t)=\Phi\left(\mathbf{x}_{s}, t\right) \cdot \tilde{a}^{A}(\mathbf{x}, t), \quad \mathbf{x} \in \Omega_{s}$

For a general function $a(x, t)$, it is not possible to tell how fast the function $\tilde{a}^{A B}(\mathbf{x}, t)$ converges towards $\tilde{a}^{B}(\mathbf{x}, t)$, when the number of scaling points is increased. However, it is clear that $\tilde{a}^{A B}(\mathbf{x}, t)=\tilde{a}^{B}(\mathbf{x}, t)$, when the number of scaling points goes to infinity. In this work, the performance of TMMC is studied in terms of practical examples.

The result of the Monte Carlo simulation is usually not the time series itself, but some scalar property derived from the time series. A typical result is the time to reach some critical value. A simplified version of the TMMC technique can be obtained, if the scaling is done for scalar numbers directly. Although the scaling would be easier to implement for the scalars than for the whole time series, the simplification has some bad properties, which are demonstrated below.

\section{RESULTS AND DISCUSSION}

\section{Approximation of Analytical Function}

The two models approximate function

$$
a(x, t)=\min \left|e^{x t}-1,0.8 \cdot\left(e^{x}-1\right)\right|, \quad t \in[0,1]
$$

The min-function was used to simulate a plateau of the time series reaching a steady state. In the model A, the analytical function was approximated by a two term Taylor series expansion. Model B output was $\tilde{a}^{B}(x, t)=a(x, t)$. The random variable $x$ was distributed uniformly between 1 and 2 . The actual outcome of the simulation, denoted by $c(x)$, was the time when $a(x, t)=a_{m}=2$ for the first time.

Figure 1 shows 100 realisations of the scalar result $c(x)$ as a function of random variable $x$. The time series scaling was used in the left side and scalar scaling on the right. At all values of $x$, model A gave higher values of $c(x)$ than model B. However, the monitoring value $a_{m}$ was not reached at all values of $x$, corresponding to a situation where the overall probability of the event is smaller than one. As a result, the scalar scaling could not be performed adequately, since there was no data to scale in the region $1.25<x<1.45$. The cumulative distributions of the 1000 realisations of $c(x)$ are shown in Fig. 2. In the regions, where the model A results do exist $(x>1.45)$, the scalar scaling worked very well, but the tail of the distribution was not corrected at all. As the number of scaling points was increased, the errors of the time series scaling converged towards zero but for the scalar scaling, the errors did not converge at all. The result shows that the time series scaling should be used if there is a possibility that the event under consideration does not take place during the simulation period. 

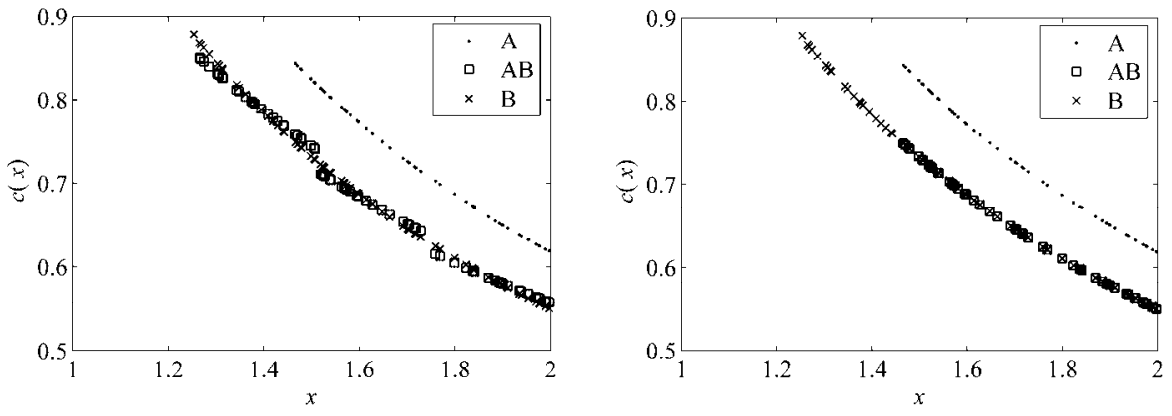

Fig. 1. Realisations of function $c(x)$ in the first example. Time series scaling on the left and scalar scaling on the right. AB is the TMMC result.
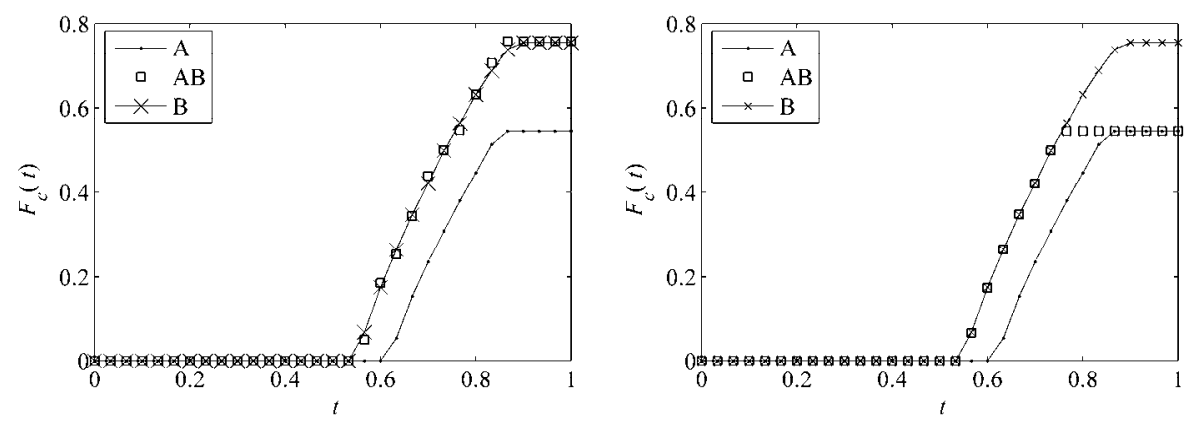

Fig. 2. Model predictions for the cumulative distribution of $c(x)$ in the first example. Time series scaling on the left and scalar scaling on the right. AB is the TMMC result.

\section{Ceiling Jet Temperature}

Two models were used to predict the ceiling jet temperature under the ceiling of a $10 \mathrm{~m} \times$ $10 \mathrm{~m} \times 5 \mathrm{~m}$ (height) room with a fire in the middle of the floor. The room had one, $2.0 \mathrm{~m}$ $\times 2.0 \mathrm{~m}$ door to ambient. The fire heat release rate was of $t^{2}$-type with a random, uniformly distributed growth time $t_{g}$. Two scalar results were studied. The scalar result $b\left(t_{g}\right)$ was the ceiling jet temperature at time $=30 \mathrm{~s}$. The scalar result $c\left(t_{g}\right)$ was the time to reach a critical temperature of $100{ }^{\circ} \mathrm{C}$ in the ceiling jet.

Alpert's ceiling jet model [7] was used as Model A and two-zone model CFAST as Model B. We simply assumed that CFAST is more accurate than Alpert's model, whether this is true or not in reality. The random space was divided into three subdomains. 1000 samples were calculated using both models. The predicted cumulative distributions of $b\left(t_{g}\right)$ are shown in the left part of Fig. 3. At all values of $t_{g}$, CFAST predicted higher temperatures than Alpert's model. TMMC distribution was very close to the CFAST result, but had small discontinuities at the boundaries of the divisions. The right hand side of Fig. 3 shows the cumulative distributions of $c\left(t_{g}\right)$. As can be seen, TMMC scaling very accurately captured the shape of the CFAST distribution. 

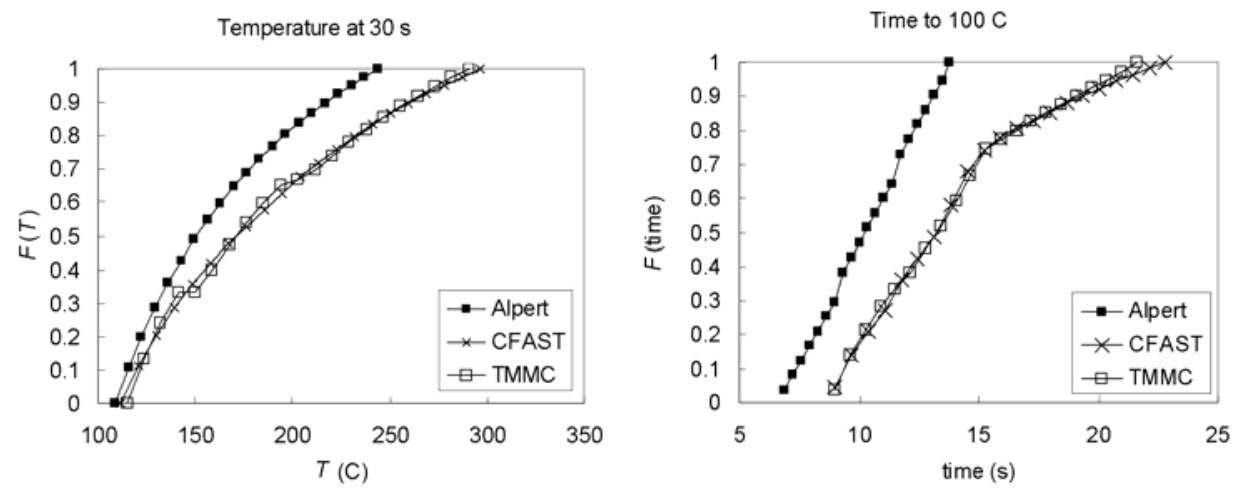

Fig. 3. Distributions of temperature at time $=30 \mathrm{~s}$ (left) and time to reach $100{ }^{\circ} \mathrm{C}$ (right).

In this example, TMMC was able to accurately reproduce the results as a full CFAST Monte Carlo, within a fraction of time required for the full CFAST Monte Carlo. Assuming that the execution times for of Alpert's model and CFAST are one and $10 \mathrm{CPU}$ seconds, respectively, and that the scaling overhead time is very small, the TMMC simulation time would be 1030 seconds in total. For comparison, the full CFAST Monte Carlo would take 10,000 seconds (2.8 hours).

\section{Simple Room Fire}

The purpose of this example was to provide a more realistic test for the TMMC technique. CFAST and FDS models were used for the fire modelling. For the evaluation of the TMMC results, a full Monte Carlo using FDS model was needed. Therefore, the size of the room was chosen very small in order to keep the simulation times short. The room was $4.0 \mathrm{~m}$ deep, $3.0 \mathrm{~m}$ wide and $3.0 \mathrm{~m}$ high, having a $1.0 \mathrm{~m}$ wide and $2.1 \mathrm{~m}$ high door to ambient. All the room boundaries were concrete and there was a concrete beam under the ceiling, $1.5 \mathrm{~m}$ from the back wall. The height of the beam was a random variable, ranging from zero to $0.6 \mathrm{~m}$. A schematic picture of the room is shown in Fig. 4 .

The fire source was a rectangular burner at the floor level. The co-ordinates and surface area of the fire source were random variables. The maximum value of the HRR per unit area was fixed to $700 \mathrm{~kW} / \mathrm{m}^{2}$. In the beginning, the heat release rate increased proportional to $t^{2}$ reaching the final value at time $t_{g}$, which was a uniformly distributed random variable. A list of the random variables is given in Table 1. The target functions were the gas temperature and heat detector activation time under the ceiling, left from the concrete beam. For gas temperature, the time to reach $200{ }^{\circ} \mathrm{C}$ was monitored. 


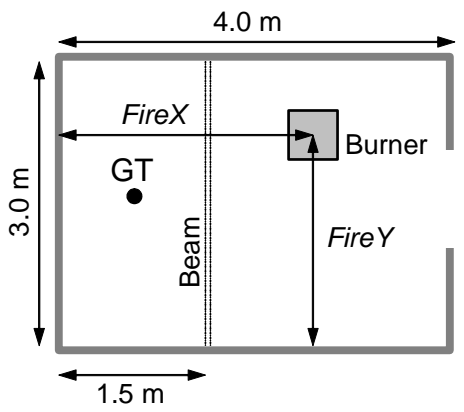

Fig. 4. The geometry of the room fire scenario. GT shows the location of the heat detector and the gas temperature measurement point $5 \mathrm{~cm}$ under the ceiling.

CFAST was used as the model A and FDS as the model B. The absolute accuracy of these codes is not discussed here. It was simply assumed, that the FDS results were more accurate than the CFAST results. In CFAST, two virtual rooms were used by splitting the room at the beam location. In FDS, a constant grid cell size of $0.10 \mathrm{~m}$ was used. Before the actual TMMC application, simulations using only CFAST and only FDS were carried out. With 1000 realisations with both models, the final distributions were well converged. The maximum difference between the cumulative distributions after 500 iterations and 1000 iterations was $0.015(1.5 \%)$. The difference was smallest in the tails of the distributions, being order of $0.001(0.1 \%)$.

Table 1. A list of random variables in the room fire example.

\begin{tabular}{|l|c|c|c|c|c|c|}
\hline Variable & Units & Distribution & Min & Max & Mean & Std.dev \\
\hline BeamHeight $z_{B}$ & $\mathrm{~m}$ & Uniform & 0.0 & 0.6 & & \\
\hline GrowthTime $t_{g}$ & $\mathrm{~s}$ & Uniform & 60.0 & 180.0 & & \\
\hline Area & $\mathrm{m}^{2}$ & Normal & 0.2 & 1.5 & 0.80 & 0.60 \\
\hline FireX & $\mathrm{m}$ & Uniform & 0.0 & 4.0 & & \\
\hline FireY & $\mathrm{m}$ & Uniform & 0.0 & 3.0 & & \\
\hline
\end{tabular}

The effect of the number of TMMC scaling points was studied by using different ways to divide the random space. The number of scaling points varied from one to 32. A summary of the different versions is given in Table 2. The basis for the division was taken from the CFAST simulations, which predicted that the fire surface area, the HRR growth time, and FireX-position were the most important random variables. Due to the division to the virtual rooms, FireX variable was especially interesting as the results had a clear discontinuity at FireX $=1.5 \mathrm{~m}$. Therefore, more divisions were used for FireX than for other variables in some of the cases. 
Table 2. A summary of scaling divisions in different TMMC versions.

\begin{tabular}{|l|c|c|c|c|c|c|}
\hline Name & $\boldsymbol{N}_{\text {tot }}$ & $\boldsymbol{N}_{\left(\mathbf{z}_{\boldsymbol{B}}\right)}$ & $\boldsymbol{N ( \boldsymbol { t } _ { \boldsymbol { g } } )}$ & $\boldsymbol{N}$ (Area) & $\boldsymbol{N ( F i r e X )}$ & $\boldsymbol{N ( F i r e Y )}$ \\
\hline TMMC(1) & 1 & 1 & 1 & 1 & 1 & 1 \\
\hline TMMC(3) & 3 & 1 & 1 & 1 & 3 & 1 \\
\hline TMMC(6) & 6 & 1 & 2 & 1 & 3 & 1 \\
\hline TMMC(27) & 27 & 1 & 3 & 3 & 3 & 1 \\
\hline TMMC(32) & 32 & 1 & 4 & 4 & 2 & 1 \\
\hline TMMC(32B) & 32 & 2 & 2 & 2 & 2 & 2 \\
\hline
\end{tabular}

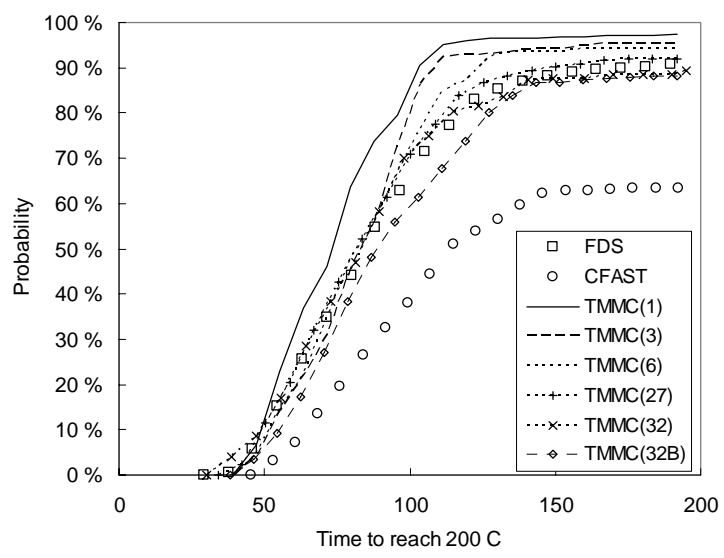

Fig. 5. Predicted probability distributions of time to reach $200{ }^{\circ} \mathrm{C}$.

A comparison of predicted probability distributions for the time when the gas temperature reached $200{ }^{\circ} \mathrm{C}$ is shown in Fig. 5. The large difference in the distributions of CFAST and FDS codes made this a challenging problem for the TMMC scaling. The overall probability was $63.5 \%$ according to CFAST, while the FDS results lead to a final probability of $90.7 \%$. Unfortunately, the simulation time was slightly too short for FDS distribution to reach a fully converged value. Therefore, an uncertainty of $1 \%$ percentage unit is associated with the final probability given by FDS.

The division of the random space had a clear effect on the accuracy of the TMMC distribution. If the division was made based on the information of the relative importance of the random variables, the higher number of scaling points generally improved the accuracy. However, if the scaling points were chosen without any prior information of the importance, the results did not improve as much as one might have expected, as was shown in the case TMMC(32B). In addition, the smoothness of the transient data affected the quality of the results. This is demonstrated in Fig. 6 showing the errors in the final probability in two cases. In the upper curve, the FDS data was not smoothed before the calculation of the scaling function $\Phi\left(\mathbf{x}_{s}, t\right)$ and in the lower curve a 5-point running average was taken. The original FDS data was saved with 2.0 second intervals. In the case of 32 scaling points, the filtered FDS data, which mostly works better, gave higher error than the unfiltered. The reason for this is currently not known. 


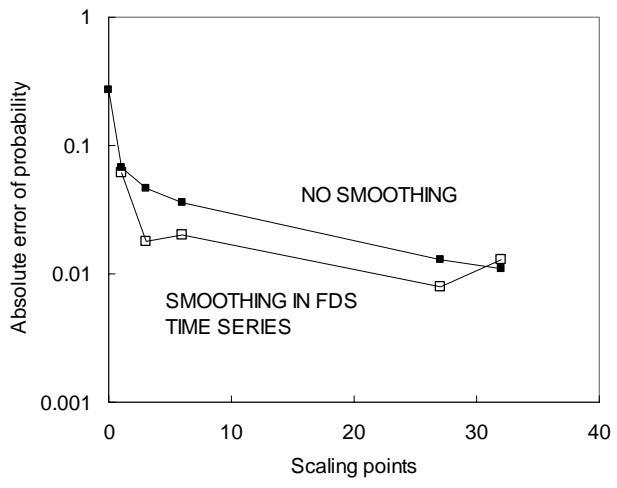

Fig. 6. The error of final probability as a function of number of scaling points.

The dependence of the TMMC accuracy on the final probability was studied by varying the critical gas temperature from $150{ }^{\circ} \mathrm{C}$ to $500{ }^{\circ} \mathrm{C}$. Fig. 7 shows the predicted final probabilities as a function of the probability given by FDS. The lowest probabilities correspond to the highest values of the critical gas temperature. The correct result is at the diagonal. The TMMC probabilities corresponding to 6, 27 and 32 scaling points are very close to the diagonal but the uniform distribution of scaling points (case 32B) results in clearly lower probabilities. An important observation of the figure is that, while CFAST did not observe the highest temperatures at all, after the scaling these highest temperatures are found, leading to non-zero probabilities in the low left corner of the figure. The accuracy of the smallest probabilities is sensitive to the smoothing of the FDS data. Here, the FDS results were obtained from the "raw" data without any smoothing, but five point smoothing was used before the computation of the scaling functions.

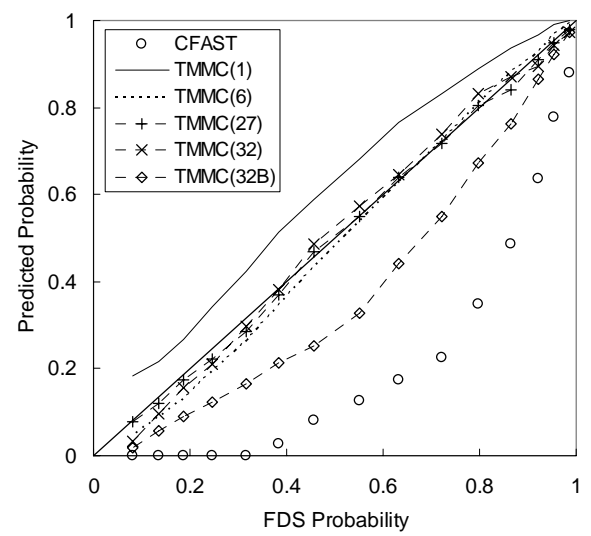

Fig. 7. The effect of the final probability on the TMMC accuracy.

TMMC scaling also improves the predicted sensitivity measures. Fig. 8 shows the predicted rank order correlation coefficients between the time to reach $200{ }^{\circ} \mathrm{C}$ temperature and the random variables. For most variables, all the three methods, CFAST, FDS and TMMC, gave very similar coefficients. However, for the HRR growth time, 
CFAST gave much lower RCC than FDS, but the TMMC result was very close to FDS result. The case with 27 scaling points was used.

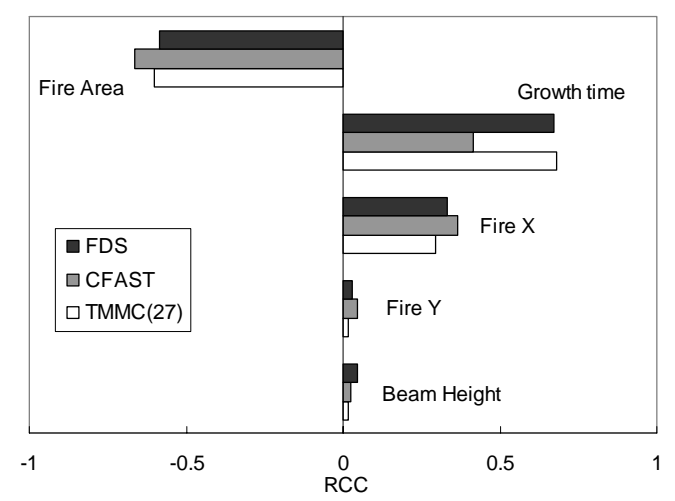

Fig. 8. Rank order correlation coefficients for the gas temperature reaching time.

For the prediction of the heat detector activation time, the TMMC method was not as successful as for the time to reach a certain gas temperature. The predicted probability distributions are shown in Fig. 9. As before, the increased number of scaling points improved the results, but all the TMMC curves fall on the original CFAST curve at high values of activation time. The reason for this turned out to be the fact that, in FDS the detector temperature was not updated after the detector activation. To be compatible with FDS, the CFAST results were also limited to the detection temperature after the detection, although CFAST actually did update the detector temperature. This limiting process forced all the scaling functions to unity in the end of the time period, which is clearly seen in Fig. 9. The conclusion is that for the TMMC method to work, the time series should not have unphysical limitations, which prevent the calculation of realistic scaling functions.

This example demonstrates that the models A and B must always represent the same physical problem, at least quantitatively. If there is no correlation between the outputs of the two models, it does not make sense to scale one with another. Unfortunately, there is no simple way to identify the cases, where this is not the case, without plotting a large number of results to the same figure, like in Fig. 1. This, in turn, would require a large number of simulation runs. A good understanding of the behaviour of the physical models is therefore required for the judgement of the applicability of TMMC technique to the problem under consideration. Additionally, a special attention should be paid to the choice of the model $\mathrm{B}$, since there is an inherent assumption that it is always more accurate than model A. 


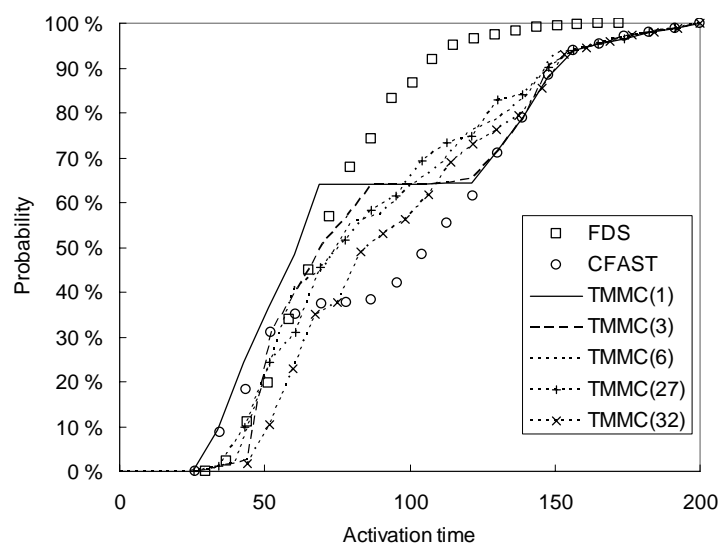

Fig. 9. Predicted probability distributions of the heat detector activation time.

\section{CONCLUSIONS}

Probabilistic Fire Simulator is a tool for Monte Carlo simulations of fire scenarios. The tool is implemented as a worksheet computing tool, and can be used as user interface for various fire models. The Monte Carlo simulations can provide the distributions of the output variables and their sensitivities to the input variables. Typical outputs are for example the times of component failure, fire detection, and flashover.

A new technique was developed that can be used to improve the accuracy of the Monte Carlo simulation. Two-model Monte Carlo is a computationally affordable technique to utilize advanced simulation techniques like CFD in the probabilistic safety assessment of large systems. In practical applications, the results of the simple but defective simulation models can be corrected by scaling them with the results achieved from an order of few tens of simulations with the more advanced model. A good accuracy can be achieved if the existing information on the relative importance of the random variables is used to efficiently place the scaling points. If such information is not available, or is not reliable, the random space must be divided uniformly in all dimensions, and the number of required scaling points may become very high. A special care must be taken to ensure that the physical time series, that are used to calculate the scaling functions, have no artificial and unphysical limitations. Such limitations may prevent the convergence of the TMMC distributions towards the correct results.

The new model has already found applications in the computation of the fire induced damage probabilities in large and complicated compartments like the switchgear rooms of the nuclear power plants. In these applications, we have demonstrated that an alternative for the use of two different computer codes is the modelling of the same scenario with one code but two different discretizations. The role of the TMMC scaling can then be seen as an utilisation of the posteriori error information. In the projects currently going on, the TMMC simulations are performed using FDS at two different grids.

As the experimental results from some 1000 experiments are not available for any real fire scenario, the only possible means to validate the probabilistic simulation technique is 
the use of the fire statistics or long series of small or medium scale laboratory experiments. The future work will include the collection of quantitative fire loss statistics and an attempt to reproduce the statistics using Monte Carlo simulation.

\section{ACKNOWLEDGEMENTS}

This study has been financed by the Finnish Centre for Radiation and Nuclear Safety, the

Ministry of Trade and Industry, Fortum Engineering Ltd. and TeollisuudenVoima Oy.

\section{REFERENCES}

[1] Metropolis, N. and Ulam, S., "The Monte Carlo Method," Journal of the American Statistical Association, 44 (1949) 247, pp.335-341.

[2] Hostikka, S. and Keski-Rahkonen, O., "Probabilistic Simulation of Fire Scenarios,” Nuclear engineering and design, 224 (2003) 3, pp. 301-311.

[3] Jones, W.W., Forney, G.P., Peacock, R.D., and Reneke, P.A. "Technical Reference for CFAST: An Engineering Tool for Estimating Fire and Smoke Transport," National Institute of Standards and Technology NIST TN 1431, Gaithersburg, MD, 2000, 190 p.

[4] McGrattan, K.B., Baum, H.R., Rehm, R.G., Hamins, A., Forney, G.P, Floyd, J.E, Hostikka, S., and Prasad, K., "Fire Dynamics Simulator (Version 3) - Technical Reference Guide," National Institute of Standards and Technology, Gaithersburg, MD, 2002, 46 p. NISTIR 6783, 2002 Ed.

[5] McKay, M.D., Beckman, R.J., and Conover, W.J., “A Comparison of Three Methods for Selecting Values of Input Variables in the Analysis of Output from a Computer Code,” Technometrics, 21(2) (1979), pp. 239-245.

[6] Hofer, E., "Sensitivity Analysis in the Context of Uncertainty Analysis for Computationally Intensive Models," Computer Physics Communications 117 (1999), pp. 1-34.

[7] Drysdale, D. An Introduction to Fire Dynamics. Wiley-Interscience, Chichester, 1999. Second Edition, p. 140. 